\title{
Paralysie cérébrale et responsabilité obstétricale
}

\section{Cerebral palsy and obstetric responsability}

\author{
C. Racinet $\cdot$ C. Cans \\ (C) Springer-Verlag France 2010
}

Le cerveau du foetus et du nouveau-né peut être la cible de diverses atteintes pathologiques (malformations, affections génétiques ou métaboliques, infections, hypoxo-ischémie, traumatisme...) qui, en cas de survie, vont laisser parfois des séquelles neurologiques dont la paralysie cérébrale (PC) est l'exemple le plus connu, car faisant l'objet de nombreuses recherches épidémiologiques et de mises en cause de la responsabilité obstétricale.

\section{Définition de la PC}

La PC est un terme d'usage universel qui inclut les appellations françaises d'infirmité motrice cérébrale (sans troubles intellectuels associés) ou IMC et d'infirmité motrice d'origine cérébrale ou IMOC.

La PC de l'enfant est un syndrome complexe aux multiples aspects cliniques et étiologiques, associé à divers facteurs de risque, et dont la définition a été souvent révisée. La définition la plus actuelle a été proposée par Rosenbaum et al. en 2007 [1] : c'est un groupe de troubles du développement de la motricité (des mouvements et des postures), entraînant une limitation de l'activité, qui sont attribués à des perturbations non progressives qui surviennent lors du développement du cerveau fœtal ou infantile. Les troubles moteurs sont souvent accompagnés de troubles sensitifs, cognitifs, de la communication, de la perception et/ou du comportement, et/ou de crises épileptiques.

La gravité des troubles observés est variable mais toujours sévère, pouvant confiner l'enfant dans un véritable

\author{
C. Racinet $(\bowtie)$ \\ UJF Grenoble, registre du handicap de l'enfant et observatoire \\ périnatal, 23, boulevard Albert-1er-de-Belgique, \\ F-38000 Grenoble, France \\ e-mail : Claude.racinet@orange.fr \\ C. Cans \\ Pôle santé publique, RHEOP-THEMAS, \\ CHU de Grenoble, BP217, \\ F-38043 Grenoble cedex 09, France
}

état grabataire avec toutes les conséquences familiales et sociales que cet état induit.

Le coût économique de l'affection est énorme, et il est donc compréhensible que les parents sollicitent une aide matérielle à la hauteur de leurs besoins. Celle-ci est actuellement plus sûrement obtenue par la recherche d'une responsabilité obstétricale, historiquement évoquée par Little dès 1862 [2].

\section{Contexte médicolégal}

Il est de fait que la responsabilité d'une mauvaise pratique obstétricale lors de l'accouchement est régulièrement recherchée et souvent retenue par les experts et les magistrats et, étant supposée être à l'origine d'une " souffrance fotale aiguë $»^{1}$ puis de lésions cérébrales à l'origine de la $\mathrm{PC}$, elle peut entraîner des indemnités qui atteignent parfois $8 \mathrm{M} €$ (sans tenir compte de leur évolution à la hausse du fait de l'inflation).

Cette situation médicolégale est relativement récente et n'est pas sans poser des problèmes tout particulièrement aux professionnels libéraux de l'obstétrique, dont le désengagement des salles d'accouchement est une réponse de plus en plus fréquente. Ceux-ci subissent une augmentation quasi

\footnotetext{
${ }^{1}$ Il faut souligner que la confusion continue de régner du fait de l'utilisation persistante - y compris dans des rapports d'expertise du concept de « souffrance fotale aiguë » (SFA), pourtant supprimé de la nomenclature des maladies depuis 1998 (CIM 9). Ce terme était estimé trop imprécis et conduisait à des diagnostics par excès du fait de l'assimilation abusive avec le concept beaucoup plus restrictif et plus précis d'asphyxie aiguë perpartum. Cette utilisation du terme de SFA par les praticiens était - et malheureusement est encore souvent - la règle devant la moindre anomalie cardiotocographique de l'état foetal lors de l'accouchement, voire de l'état de l'enfant à la naissance. Elle équivaut à la reconnaissance implicite de la responsabilité obstétricale en cas de problèmes neurologiques postnatals, et le diagnostic suggéré par le terme SFA d'asphyxie fotale aiguë suit définitivement l'enfant dans son carnet de santé, sans remise en cause d'une origine présumée asphyxique, même en l'absence des critères objectifs définis par le consensus ACOG-AAP de 2003, repris en France dans les recommandations du CNGOF pour la surveillance fotale [3].
} 
exponentielle de leurs primes d'assurance, mais aussi vivent dans l'angoisse permanente d'être potentiellement à l'origine d'une asphyxie foetale perpartum, qui demeure la cause prioritairement évoquée par les demandeurs (les parents) en cas de PC chez leur enfant.

Aux États-Unis, une enquête faite en 2006 par le Collège américain des gynécologues-obstétriciens (ACOG) a révélé que $89,2 \%$ des répondants au nombre de 10869 ont été confrontés à une procédure judiciaire concernant leur responsabilité professionnelle avec une moyenne de 2,62 plaintes par médecin [4].

En France, les statistiques du Syndicat national des gynécologues-accoucheurs (Syngof) révèlent un désengagement régulier de la pratique obstétricale libérale (40 arrêts par an environ depuis 2007, $61 \%$ des libéraux ne font plus d'activité d'obstétrique en 2009) [5].

Ce sont surtout les problèmes liés à la PC qui sont en cause, liés à certaines expertises à charge impliquant régulièrement la responsabilité d'une asphyxie aiguë et le caractère évitable de la $\mathrm{PC}$ : ces opinions expertales ne sont généralement pas basées sur des publications peer-reviewed faisant état de travaux de recherche convaincants [6].

Une autre conséquence de cette évolution est la dérive vers une médecine défensive avec une inflation croissante des indications de césarienne, notamment pour des anomalies du rythme cardiaque foetal (RCF) sur le monitorage perpartum dont on connaît pourtant le manque flagrant de spécificité. Outre le fait que la principale méta-analyse des essais randomisés comparant monitorage électronique et auscultation clinique n'a pas révélé de rôle protecteur du monitorage pour la PC [7], cette dérive a une contrepartie maternelle, à savoir une augmentation des anomalies placentaires source d'hémorragies gravissimes lors des grossesses suivantes et de mortalité maternelle (en France, $50 \%$ des morts maternelles sont évitables, soit 40 cas par an).

\section{Données épidémiologiques}

\section{Évolution de la prévalence de la PC}

Alors que la mortalité périnatale a rapidement chuté en France après le Plan périnatalité de 1971, on constate de façon a priori surprenante que le taux global (tous âges gestationnels confondus) de PC est resté sans variation significative au fil du temps... De fait, le constat de cette stabilité du taux de PC est général et touche tous les pays développés ou en développement où la surveillance du travail n'est souvent pas optimale, ce qui sous-entend que la qualité des soins périnatals intervient très peu dans la prévalence des $\mathrm{PC}$ [8] et que ce marqueur n'est pas un bon marqueur de cette qualité.

En France, les données en population du registre du handicap de l'enfant et observatoire périnatal (RHEOP)

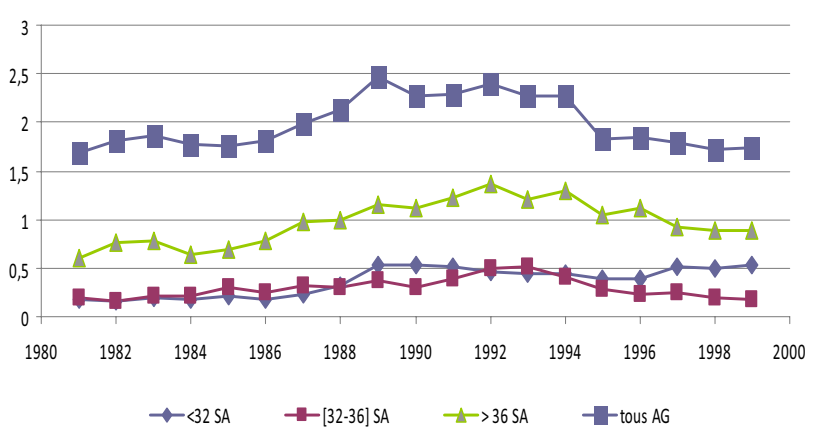

Fig. 1 Évolution de la prévalence de la paralysie cérébrale en Isère (France) de 1980 à 2000 (moyenne mobile sur 3 ans - taux pour 1000 naissances vivantes) - (Données de RHEOP)

démontrent une stabilité de la prévalence globale allant, respectivement, de 1,6 à $1,7 \%$ pour les enfants nés en 1980 et en 2000 [9] (Fig. 1), sans variation significative en fonction de l'âge gestationnel. Les données de surveillance of cerebral palsy in Europe (SCPE) [groupe collaboratif qui inclut le RHEOP qui héberge la base de données commune] confirment avec une puissance décuplée que la prévalence de la PC pour les enfants nés vivants de plus de $2500 \mathrm{~g}$ varie pour les années 1980 et 1996 de 1,16 à $0,99 \%$ o $(p=0,14, \mathrm{NS})$ [10]. Mais contrairement à ce qui était constaté avant les années 1980, la prévalence chez les très petits poids de moins de $1500 \mathrm{~g}$ (dont la fréquence a augmenté de 0,5 à presque $1 \%$ ) s'est réduite de 60,6 à $39,5 \%$, ce qui conduit à un taux absolu légèrement accru alors que la survie des très petits poids a doublé [11].

Clark et Hankins [8] ont démontré que cette stabilité du taux global de PC ne résultait pas d'un équilibre entre une baisse chez les enfants nés à terme (par l'effet supposé mais jamais démontré du rôle préventif du RCF sur la survenue d'une PC [7]) et une augmentation des PC chez les prématurés du fait de leur meilleure survie, mais d'une réelle stabilité de tous les taux. Depuis, les analyses des données du réseau SCPE ont confirmé que la prévalence est stable à long terme chez les enfants de plus de $2500 \mathrm{~g}$ et que, pour les enfants de moins de $1500 \mathrm{~g}$, même si leur taux d'exposition augmente, ce groupe d'enfants ne représente qu'un quart des enfants avec PC.

\section{Évolution de l'asphyxie périnatale et de l'encéphalopathie néonatale}

Il s'agit de deux précurseurs indispensables pour retenir une cause asphyxique à l'origine d'une PC.

L'asphyxie périnatale avait de tout temps été pointée comme étant une importante cause de mortalité et morbidité périnatales. Dans le rapport 2009 du RHEOP, on constate 
que la mortinatalité spontanée est en fait restée stable allant de 3,4\%o naissances en 1988 à 3,7\%o en 2008 [9], mais que la part des décès perpartum après $27 \mathrm{SA}$ a chuté de façon très significative de 23 à $6 \%$.

L'étude en population faite en Californie sur plus de 5000000 de nouveau-nés, de 1991 à 2000, a porté sur les cas d'asphyxie à la naissance et a démontré une réduction drastique de $91 \%$, le taux passant de 14,8 à 1,3\%o naissances vivantes. Ce résultat suggère une implication restreinte de cet item dans la genèse d'une PC dont le taux est resté stable en Californie pendant la même période [12].

L'encéphalopathie néonatale, intermédiaire obligatoire entre asphyxie fœtale avérée et $\mathrm{PC}$, a longtemps été qualifiée par le terme hypoxo-ischémique (EHI), sa genèse étant historiquement considérée d'origine asphyxique quasi exclusive. Graham et al. ont estimé que, dans une méta-analyse observationnelle colligeant 386 enfants dont le $\mathrm{pH}$ néonatal était inférieur à 7,00, on observait 17,2\% d'encéphalopathie néonatale présumée postasphyxique mais sur des critères très hétérogènes et de spécificité discutable [17]. Les données ne permettent aucune hypothèse sur l'évolution dans le temps. Or, la baisse régulière de l'EHI a été constatée par Smith et al. [13] dans une population homogène de 75000 enfants nés à terme de 1976 à 1996 en Grande-Bretagne : le taux s'abaisse de 7,7 à 4,6\%, puis à 1,9\%o naissances vivantes, alors que le chiffre de PC après EHI est respectivement de deux cas, un cas et deux cas.

De plus, des études en population basées sur des critères standardisés suggèrent que l'association $\mathrm{PC}$-asphyxie fœtale aiguë perpartum n'est pas aussi fréquente qu'on le croyait et qu'elle se situerait à un niveau inférieur à $5 \%[14,15]$.

\section{Évolution des pratiques obstétricales}

La prévalence de la PC est donc restée sans variation significative au fil du temps. Et cela, malgré la quasigénéralisation du monitorage fœetal perpartum et une augmentation spectaculaire du taux de césarienne passé de $5 \%$ dans les années 1970 à plus de $20 \%$ en 2008 dans les pays occidentaux développés [8].

En Isère, l'enregistrement des césariennes par la PMI existe depuis 1989 et celui des indications maternelles ou fœtales depuis 1996 (Tableau 1). Entre 1989 et 2000, on assiste à une augmentation des césariennes de 59,6\%, alors que le taux d'enfants nés par césarienne pour indication fœtale atteint 7,8 \% en 2000 (l'hypothèse d'anomalies fréquentes du RCF est plausible). Bien entendu, le monitorage comme l'auscultation peuvent dépister la survenue de rares catastrophes obstétricales juste avant leur traduction clinique et permettre le sauvetage fœtal par une extraction en urgence.

Le plus souvent, la césarienne est pratiquée pour des anomalies du RCF pendant le travail selon l'hypothèse physiopathologique émise par HON en 1975 que le monitorage devrait permettre de prendre les décisions nécessaires estimées pouvoir réduire de moitié le risque de PC : la méta-analyse de Graham n'a pas confirmé cette hypothèse.

Il est possible, comme l'ont réalisé Clark et Hankins pour les États-Unis [8], de calculer le retentissement démographique en France des évolutions des données de prévalence et de pratiques à partir du modèle du RHEOP. On considère qu'il y a 800000 naissances et 1600 cas de PC dont 400 au maximum sont d'origine postasphyxique (chronique et aiguë). L'évolution de la prévalence de la $\mathrm{PC}$ des très petits poids permet d'estimer qu'en 2000 on peut s'attendre à une augmentation très discrète de 80 cas de PC (chiffre obtenu par le croisement de la baisse de la prévalence par l'augmentation du taux d'exposition au terme de la période de 20 ans s'achevant en 2000). Mais parallèlement, l'augmentation très nette des césariennes, si elle devait s'avérer efficace pour prévenir la $\mathrm{PC}$ dans $50 \%$ des cas, selon l'hypothèse évoquée plus haut, serait amplement suffisante pour éradiquer totalement la PC. En effet, un calcul arrondi estime en 2000 le nombre de césariennes pour indication fœtale à 62 400, ce qui serait a priori susceptible d'éviter 31000 cas (chiffre très supérieur au chiffre observé de 1600 cas, toutes causes confondues) ! Même si son efficacité neuroprotectrice n'était que de $5 \%$, le niveau atteint par les césariennes d'indication fœtale aboutirait à un nombre évité de PC de 3100 cas, alors que le chiffre attendu de PC postasphyxique est de 400 cas ! On peut estimer que l'éradication des PC postasphyxiques, en retenant l'estimation la plus large, serait obtenue même si des actions obstétricales pertinentes et efficaces ne se produisaient que dans $0,6 \%$ des accouchements avec anomalies du RCF, ce qui impliquerait que la vigilance obstétricale est prise en défaut dans plus de $99 \%$ des cas (hypothèse peu crédible) ! Par ailleurs, quelle valeur attribuer à une intervention induite

Tableau 1 Évolution des taux (\%) de césarienne en Isère de 1989 à 2000 et de la part liée à des indications fœtales de 1996 à 2000 (données du CS8-PMI Isère)

\begin{tabular}{|lllllllllllll|}
\hline Années & $\mathbf{1 9 8 9}$ & $\mathbf{1 9 9 0}$ & $\mathbf{1 9 9 1}$ & $\mathbf{1 9 9 2}$ & $\mathbf{1 9 9 3}$ & $\mathbf{1 9 9 4}$ & $\mathbf{1 9 9 5}$ & $\mathbf{1 9 9 6}$ & $\mathbf{1 9 9 7}$ & $\mathbf{1 9 9 8}$ & $\mathbf{1 9 9 9}$ & $\mathbf{2 0 0 0}$ \\
\hline Taux CS & 10,9 & 11,8 & 12,2 & 12,5 & 13,2 & 13,3 & 12,8 & 14,8 & 15,7 & 16,2 & 17,3 & 17,4 \\
Indications fœtales & - & - & - & - & - & - & - & 5,8 & 6,6 & 6,8 & 7,7 & 7,8 \\
\hline
\end{tabular}


par un RCF anormal et dont l'efficacité préventive sur la PC serait aussi faible, les risques maternels étant alors indiscutablement plus importants que le bénéfice neurologique fœtal espéré ?...

En résumé, on surveille de mieux en mieux les accouchements par le monitorage fœtal, on fait - en partie de ce fait - de plus en plus de césariennes, on observe certes une amélioration de la morbidité perpartum et néonatale, mais on a toujours autant de PC... Cette stabilité désespérante permet d'évoquer le fait que la PC, sauf quelques exceptions, est essentiellement une pathologie liée au développement, d'autant que nos efforts de prévention sont très majoritairement mis en échec ou compensés par l'augmentation des facteurs d'exposition (dans le cas des petits poids de naissance).

\section{L'étiologie de la PC est majoritairement d'origine pergravidique}

Dans la plupart des cas de PC, il existe des facteurs de risques majeurs associés à la neuropathologie tels qu'une infection intra-utérine, un accouchement prématuré, une restriction de croissance fotale intra-utérine, des complications de grossesses multiples, une hémorragie ante-partum et des anomalies neurologiques congénitales. Il a été plus récemment souligné le rôle de facteurs anténatals souvent silencieux qui sont associés à une $\mathrm{PC}$, tels qu'une thrombophilie héréditaire, un polymorphisme des cytokines et une exposition virale dans la période périnatale. D'autres facteurs tels qu'une pyrexie pendant l'accouchement ont été également fortement associés à la survenue d'une PC. Ce dernier facteur peut refléter une infection intra-utérine précoce qui a ou n'a pas entraîné de chorioamniotite. L'infection intra-utérine ou néonatale, survenant spécialement sur le cerveau très vulnérable d'un enfant prématuré, peut initier une réponse inflammatoire qui attaque les neurones en développement. Il y a aussi probablement de nombreux cofacteurs qui ne sont pas encore bien compris et qui prédisposent certains fœetus ou nouveau-nés à un processus destructif évolutif qui peut conduire à la neuropathologie d'une $\mathrm{PC}$ au-delà de la période périnatale.

\section{Conclusion}

La PC reste un sujet préoccupant du fait de sa prévalence stable, insensible aux actions qui tentent d'écourter, voire de court-circuiter le travail d'accouchement et de son contexte médicolégal qui a un impact négatif sur la démographie obstétricale. Il parait indispensable d'approfondir les recherches périnatales pour identifier les causes d'un grand nombre de cas catalogués « cause inconnue » ou par défaut « asphyxie périnatale ». L'avenir réside certainement dans une perspective de génétique clinique, dans l'identification de nouvelles pathologies par l'apport conjoint de la protéomique et de la métabolomique [16].

\section{Références}

1. Rosenbaum P, Paneth N, Leviton A, et al (2007) A report: the definition and classification of cerebral palsy April 2006. Dev Med Child Neurol Suppl 109:8-14

2. Little WJ (1861-1862) On the influence of abnormal parturition, difficult labours, premature births and asphyxia neonatorum on the mental and physical condition of the child, especially in relation to deformities. Trans Obstet Soc London 3:293

3. Racinet C, Hoffmann P (2010) Asphyxie périnatale et paralysie cérébrale : implications médicolégales. Bull Acad Natle Med $194, \mathrm{n}^{\circ} 6$ (sous presse)

4. Wilson N, Strunk A (2007) Overview of the 2006 ACOG survey on professional lability. ACOG Clin Rev 12(2):1,13-6

5. Mace JM (2010) Impact de la RCP sur les soins du point de vue économique et géographique. Les Cahiers SYNGOF, $\mathrm{n}^{\circ} 82$ (septembre 2010):19-22

6. McLennan A, Hankins G, Speer M (2008) Only an expert witness can prevent cerebral palsy ! OG 8:28-30

7. Graham E, Petersen S, Christo D, et al (2006) Intrapartum electronic fetal heart rate monitoring and the prevention of perinatal brain injury. Obstet Gynecol 108(3Pt1):656-66

8. Clark SL, Hankins GD (2003) Temporal and demographic trends in cerebral palsy-Fact and fiction. Am J Obstet Gynecol 188:628-33

9. RHEOP (2009) Rapport annuel 2009 téléchargeable sur le site : www-rheop.ujf-grenoble.fr/rheop/Accueil.htm

10. Sellier E, Surman G, Himmelmann K, et al (2010) Trends in prevalence of cerebral palsy in children born with a birthweight of 2,500 g or over in Europe from 1980 to 1999. Eur J Epidemiol $25: 635-42$

11. Platt MJ, Cans C, Johnson A, et al (2007) Trends in cerebral palsy among infants of very low birthweight $(<1500 \mathrm{~g})$ or born prematurely $(<32$ weeks) in 16 European centres: a database study. Lancet 369:43-50

12. Wu Y, Backstrand KH, Zhao S, et al (2004) Declining diagnosis of birth asphyxia in California: 1991-2000. Pediatrics 114:1584-90

13. Smith J, Wells L, Dodd K (2000) The continuing fall in incidence of hypoxic-ischemic encephalopathy in term infants. Br J Obstet Gynecol 107:461-6

14. Strijbis EM, Oudman I, Van Essen P, McLennan A (2006) Cerebral palsy and the application of the international criteria for acute intrapartum hypoxia. Obstet Gynecol 107:1357-65

15. Ettori C, Racinet C, Arnould P, et al (2009) Imputabilité de l'asphyxie fœtale perpartum dans la paralysie cérébrale infantile (cohorte d'enfants nés en Isère de 1993 à 1999). Communication orale aux $39^{\text {es }}$ Journées nationales de la SFMP, Angers. Le texte peut être demandé à claude.racinet@orange.fr

16. Spitzer AR, Chace D (2008) Proteomics- and metabolomicsbased neonatal diagnostics in assessing and managing the critically ill neonate. Clin Perinatol 35:695-716

17. Graham EM, Ruis KA, Hartman AL, et al (2008) A systematic review of the role of intrapartum hypoxia-ischemia in the causation of neonatal encephalopathy. Am J Obstet Gynecol 199(6): $587-95$ 